\title{
Diffuse flux of galactic neutrinos and gamma rays
}

\author{
J.M. Carceller, M. Masip \\ CAFPE and Departamento de Física Teórica y del Cosmos \\ Universidad de Granada, E-18071 Granada, Spain \\ E-mail: jmcarcell@correo.ugr.es, masip@ugr.es
}

\begin{abstract}
We calculate the fluxes of neutrinos and gamma rays from interactions of cosmic rays with interstellar matter in our galaxy. We use EPOS-LHC, SIBYLL and GHEISHA to parametrize the yield of these particles in proton, helium and iron collisions at kinetic energies between 1 and $10^{8} \mathrm{GeV}$, and we correlate the cosmic ray density with the mean magnetic field strength in the disk and the halo of our galaxy. We find that at $E>1 \mathrm{PeV}$ the fluxes depend very strongly on the cosmic-ray composition, whereas at 1-5 GeV the main source of uncertainty is the cosmic-ray spectrum out of the heliosphere. We show that the diffuse flux of galactic neutrinos becomes larger than the conventional atmospheric one at $E>1$ $\mathrm{PeV}$, but that at all IceCube energies it is 4 times smaller than the atmospheric flux from forward-charm decays.
\end{abstract}




\section{Contents}

1 Introduction $\quad 1$

2 Neutrinos and gammas from galactic CRs $\quad 2$

$3 \quad$ Neutrino and gamma-ray yields $\quad 4$

4 Angular dependence of the fluxes $\quad 6$

5 Neutrino and gamma-ray fluxes $\quad 8$

6 Summary and discussion $\quad 11$

\section{Introduction}

Cosmic rays (CRs) are a puzzling component of our universe. The simplicity of their spectrum between 10 and $10^{11} \mathrm{GeV}$, a power law $E^{-\alpha}$ with $\alpha \approx 2.7$, is apparent but very surprising, since it suggests that nature has used the same strategy to accelerate all of them. It also indicates that their propagation from the sources to the Earth is universal, in the sense that it scales trivially with the CR energy. Actually, both ends of the power law describing their spectrum could be mere propagation effects caused by the heliosphere (acting as a magnetic barrier at $\mathrm{CR}$ energies below $10 \mathrm{GeV}$ ) and by the $2.7 \mathrm{~K} \mathrm{CMB}$ (causing inelastic collisions above $10^{11} \mathrm{GeV}[1,2]$ ). A closer look at the spectrum (see [3] for a review), however, reveals features that may have an important physical meaning: at the $10^{6.5} \mathrm{GeV}$ knee the spectral index changes from 2.7 to 3 , whereas at the $10^{9.5} \mathrm{GeV}$ ankle it goes back to 2.7. The knee and the ankle could signal, respectively, the energy limit of the main accelerators in our galaxy and the point where most CRs have an extragalactic origin.

Another remarkable feature in the $\mathrm{CR}$ flux is its almost perfect isotropy: $\mathrm{TeV}$ CRs reach the Earth with the same frequency (at the 0.1\% level [4]) from all directions. Being charged, CRs are coupled to the galactic and intergalactic magnetic fields, and their isotropy is obtained after very long trajectories that lose memory of the position of the sources. In particular, the time of flight of $10-100 \mathrm{GeV}$ CRs can be estimated by comparing the ratio of light $(\mathrm{Li}, \mathrm{Be}, \mathrm{B})$ to medium $(\mathrm{C}, \mathrm{N}, \mathrm{O})$ nuclei in their composition [5]. Light nuclei are much more abundant in CRs than in the solar system, which reveals their origin as secondary particles produced in collisions of medium nuclei while crossing around $3 \mathrm{Mpc}$ of interstellar (IS) medium. This length, confirmed by the measurement of radioactive clock isotopes in the CR flux [6], is much larger than the $0.3 \mathrm{kpc}$ thickness or the $30 \mathrm{kpc}$ diameter of our galaxy. CRs behave like a very diffuse gas trapped in the galaxy by magnetic fields [7].

Up to energies close to the knee the main source of CRs could be galactic supernova remnants. Simple arguments suggest a spectral index at the acceleration site around $\alpha_{0} \approx 2$ independent from the charge or the mass of the CR [8]. The observed spectrum, with $\alpha=2.6-$ 2.7, would then result after including propagation effects; as more energetic CRs escape faster into the halo, their density in the galactic disk will be reduced by a factor of $\approx E^{-0.5}$ (typical for a Kraichnan spectrum of magnetic turbulences [7]). Satellite and balloon experiments have found that the $\mathrm{CR}$ composition is dominated by hydrogen and ${ }^{4} \mathrm{He}$ nuclei (at $E<E_{\text {knee }}$ 
we can neglect the presence of heavier nuclei), and that both species have slightly different spectral indexes $[9,10]$. Between 10 and $10^{6.7} \mathrm{GeV}$ we estimate

$$
\Phi_{p}=1.3\left(\frac{E}{\mathrm{GeV}}\right)^{-2.7} \text { protons } /\left(\mathrm{cm}^{2} \mathrm{~s} \mathrm{sr} \mathrm{GeV}\right)
$$

and

$$
\Phi_{\mathrm{He}}=0.54\left(\frac{E}{\mathrm{GeV}}\right)^{-2.6} \text { nuclei } /\left(\mathrm{cm}^{2} \mathrm{~s} \mathrm{sr} \mathrm{GeV}\right) .
$$

This implies an all-nucleon flux around $\Phi_{N} \approx 1.8 \times 10^{4}(E / \mathrm{GeV})^{-2.7}\left(\mathrm{~m}^{2} \mathrm{~s} \mathrm{sr} \mathrm{GeV}\right)^{-1}$ and also that the number of protons and He nuclei becomes similar at $E \approx 10 \mathrm{TeV}$. Notice that the number of helium to hydrogen nuclei in CRs is much larger than the 1 to 12 ratio obtained from Big-Bang nucleosynthesis. Between the knee and the ankle (i.e., $10^{6.7} \mathrm{GeV}<E<10^{9.5} \mathrm{GeV}$ ) the CR composition is uncertain, while the spectrum becomes

$$
\Phi=330\left(\frac{E}{\mathrm{GeV}}\right)^{-3.0} \text { nuclei } /\left(\mathrm{cm}^{2} \mathrm{~s} \mathrm{sr} \mathrm{GeV}\right) .
$$

Information about CRs comes not only from direct measurements, it can be completed by observations in other channels: CR interactions with IS matter produce a diffuse flux of secondary neutrinos and gamma rays that is sensitive to their composition and spectrum, and also to the gas distribution in our galaxy. At $\mathrm{TeV}-\mathrm{PeV}$ energies these galactic neutrinos could define a flux observable at IceCube [11], whereas lower energy gamma rays may bring information, for example, about the CR density out of the heliosphere. At any rate, these secondary particles are a certain background in the search for astrophysical sources at neutrino and gamma-ray observatories.

In this paper we will attempt a complete calculation of the neutrino and gamma-ray fluxes from CR spallation inside our galaxy [12-22], paying special attention to the energy and composition dependence of the yields. In particular, we will use the MonteCarlo simulators EPOS-LHC [23], SIBYLL [24] and (at $E<50 \mathrm{GeV}$ ) GHEISHA [25] to obtain precise parametrizations of the $\left(\gamma, \nu_{i}, \bar{\nu}_{i}\right)$ yields for proton, helium and iron primaries at different energies. We will also define a scheme where the CR density inside our galaxy is correlated with the mean magnetic field strength in the disk and the halo. This and the gas distribution will provide the angular dependence of the gamma-ray and neutrino fluxes both at IceCube and Fermi-LAT [26] energies.

\section{Neutrinos and gammas from galactic CRs}

Consider a point $\vec{r}=r \vec{u}_{r}$ in our galaxy where the CR number density for the species $A=$ $(p, \mathrm{He}, \ldots, \mathrm{Fe})$ is $n_{A}(E, \vec{r})$ and where the IS gas density is $\rho_{i}(\vec{r})$, with $i=(p, \mathrm{He})$. If the $\mathrm{CR}$ distribution is isotropic, the number density and the flux $\Phi_{A}(E, \vec{r})$ at that point are simply related:

$$
\Phi_{A}(E, \vec{r})=\frac{c}{4 \pi} n_{A}(E, \vec{r}) .
$$

Neutrinos from collisions of type $A$ CRs with type $i$ IS gas that reach the Earth (at $\vec{r}=0$ ) from direction $\vec{u}_{r}$ may be produced at any point along the line generated by $\vec{u}_{r}$. The total neutrino flux is then $[8,27]$

$$
\Phi_{\nu}\left(E, \vec{u}_{r}\right)=\sum_{A, i} \int_{0}^{\infty} \mathrm{d} r \int_{E}^{\infty} \mathrm{d} E^{\prime} \frac{1}{\lambda_{A i}\left(E^{\prime}, \vec{r}\right)} \Phi_{A}\left(E^{\prime}, \vec{r}\right) \frac{\mathrm{d} n_{A i}^{\nu}}{\mathrm{d} E}\left(E ; E^{\prime}\right)
$$


where the interaction length for $A i$ collisions at CR energy $E^{\prime}$ reads

$$
\lambda_{A i}\left(E^{\prime}, \vec{r}\right)=\frac{m_{i}}{\rho_{i}(\vec{r}) \sigma_{A i}\left(E^{\prime}\right)}
$$

and $\mathrm{d} n_{A i}^{\nu} / \mathrm{d} E$ expresses the yield of neutrinos of energy $E$ obtained in those collisions. An analogous expression including the transparency $\eta(E, \vec{r})$ of the galaxy would describe the gamma-ray flux reaching the Earth. It is revealing to rewrite Eq. (2.2) in terms of the fraction $x=E / E^{\prime}$ of energy taken by the neutrino:

$$
\Phi_{\nu}\left(E, \vec{u}_{r}\right)=\sum_{A, i} \int_{0}^{\infty} \mathrm{d} r \rho_{i}(\vec{r}) \int_{0}^{1} \mathrm{~d} x \frac{\sigma_{A i}(E / x)}{m_{i}} \Phi_{A}(E / x, \vec{r}) x^{-1} f_{A i}^{\nu}(x, E / x),
$$

with $f_{A i}^{\nu}\left(x, E^{\prime}\right)$ the yield of neutrinos carrying a fraction $x$ of the CR energy $E^{\prime}$. This equation goes into a much simpler form after several assumptions that are common in the literature (in next sections we will check their validity):

1. Since neutrinos come from pions and these are created in nucleon-nucleon collisions, one expects that the yield from a He nucleus of energy $E$ coincides with that from a proton of energy $E / 4$, i.e., the yield is a function of the energy per nucleon in the CR. This motivates the definition of a flux $\tilde{\Phi}_{C R}(E)$ of CRs per unit of energy per nucleon, ${ }^{1}$

$$
\tilde{\Phi}_{C R}(E, \vec{r})=\sum_{A} \tilde{\Phi}_{A}(E, \vec{r})=\sum_{A} A \Phi_{A}(A E, \vec{r}) .
$$

2. The spectrum of secondaries after the fragmentation of a high-energy CR does not depend on the target (the $\mathrm{H}$ or He nucleus in the IS gas).

3. One may take a constant spectral index $\alpha$ for the CR flux in the energy interval of interest.

4. The CR density is homogeneous inside the galactic disk, like in the usual leaky-box model: $\tilde{\Phi}_{C R}(E, \vec{r})=C E^{-\alpha}$. Later on we will correlate the CR flux with the magnetic fields in the halo and the disk.

5. For the cross sections fixing the interaction lengths, one may neglect their energy dependence and assume a $A^{2 / 3}$ scaling with the mass number of the target and the projectile.

After all these approximations, the sum over the different species $A$ in the CR flux and over the two components in the IS gas (around 3 grams of hydrogen per each gram of helium) gives

$$
\begin{aligned}
\Phi_{\nu}\left(E, \vec{u}_{r}\right) & =C E^{-\alpha} f \frac{\sigma_{p p}}{m_{p}} \int_{0}^{\infty} \mathrm{d} r \rho_{I S}(\vec{r}) \int_{0}^{1} \mathrm{~d} x x^{\alpha-1} f_{p p}^{\nu}(x) \\
& =\frac{C f \sigma_{p p}}{m_{p}} X\left(\vec{u}_{r}\right) Z_{\nu p}^{(\alpha-1)} E^{-\alpha}
\end{aligned}
$$

\footnotetext{
${ }^{1}$ Notice that $\tilde{\Phi}_{C R}$ is not the all-nucleon flux $\Phi_{N}(E)=\sum_{A} A^{2} \Phi_{A}(A E)$ used to calculate atmospheric lepton fluxes.
} 

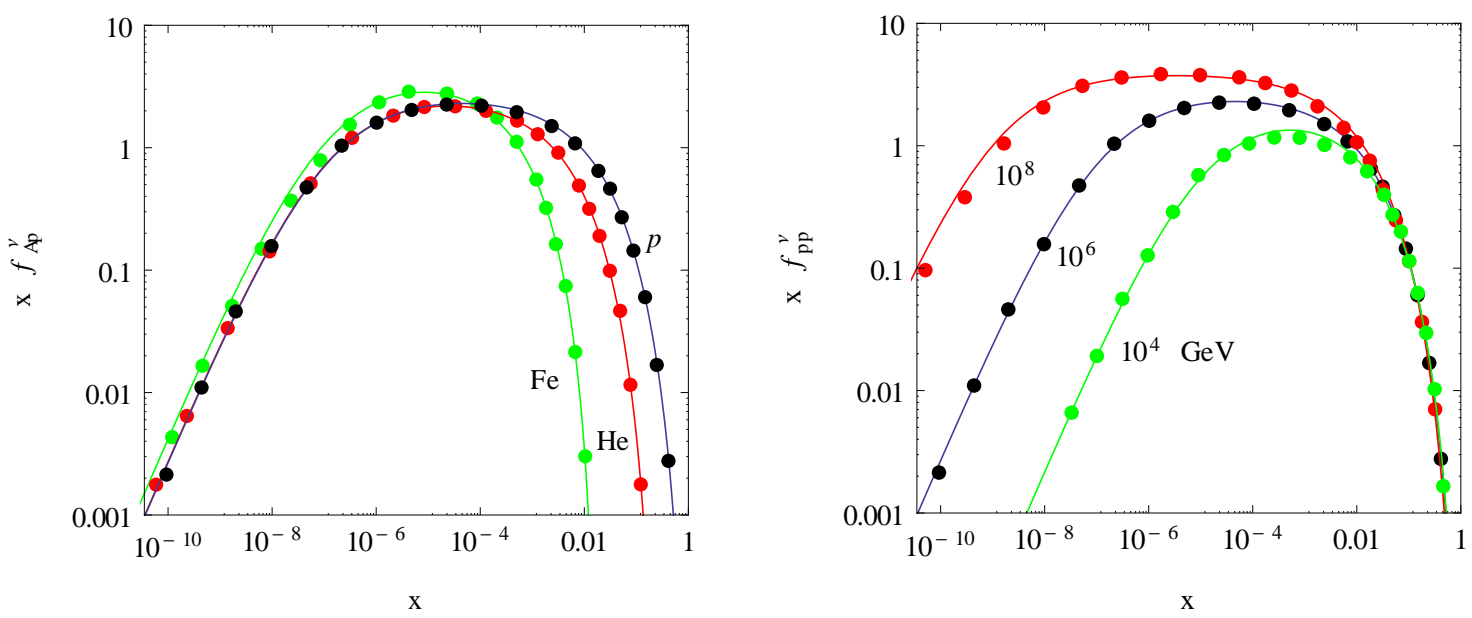

Figure 1. Left. $\nu_{e}$ yield $f_{A p}^{\nu_{e}}\left(x, 10^{6} \mathrm{GeV}\right)$ from $A=$ proton, helium and iron collisions obtained with EPOS-LHC (dots) and our 2-parameter fits (solid). Right. $f_{p p}^{\nu_{e}}(x, E)$ for $E=10^{4} \mathrm{GeV}, 10^{6} \mathrm{GeV}$ and $10^{8} \mathrm{GeV}$.

where $\rho_{I S}(\vec{r})$ is the total mass density at $\vec{r}, X\left(\vec{u}_{r}\right)$ is the total depth of IS matter along the direction $\vec{u}_{r}$, and $Z_{\nu p}^{(\alpha-1)}$ is the $(\alpha-1)$-moment of the neutrino yield in $p p$ collisions. The parameter

$$
f=\left(\frac{3+4^{-1 / 3}}{4}\right) \sum_{A} r_{A} A^{\frac{2}{3}},
$$

with $r_{A}$ the fraction of the primary $A$ in the total flux given by Eq. (2.5) $\left(r_{p} \approx 0.9\right.$ and $r_{\mathrm{He}} \approx 0.1$ for the fluxes in Eqs. $\left.(1.1,1.2)\right)$, takes into account the mixed composition both of the IS gas and of the CR flux.

The expression in Eq. (2.6) implies neutrino and gamma-ray fluxes with the same spectral index as the primary CRs and proportional both to the total depth (column density) along each direction in our galaxy and to the order-1.7 $Z$-moment of the yield in $p p$ collisions. Let us use MonteCarlo simulators to analize in detail cross sections and yields and establish the validity of these approximations at different energies.

\section{$3 \quad$ Neutrino and gamma-ray yields}

We plot in Fig. 1-left the $\nu_{e}$ yield from the collision of a $10^{6} \mathrm{GeV}$ proton $(A=1)$, $\mathrm{He}(A=4)$ or $\mathrm{Fe}(A=56)$ with a proton at rest. We have obtained these average yields after the simulation of $10^{4}$ events with EPOS-LHC. In the plot we include a 2-parameter fit (solid line) of type

$$
f_{A p}^{\nu}(x, E)=a \frac{(1-A x)^{3}}{x} \frac{e^{-b(A x)^{0.43}}}{\left(1+\sqrt{\frac{0.1 \mathrm{GeV}}{x E}}\right)^{2}},
$$

with $a(E, A)$ and $b(E, A)$ the energy and mass-number dependent parameters. In Fig 1-right we explore how these yields change with the energy. For the gamma yield we use the same 


\begin{tabular}{c|c|c|c|c|}
\multicolumn{1}{c}{} & \multicolumn{1}{c}{$\nu_{e}$} & $\bar{\nu}_{e}$ & $\nu_{\mu}$ & $\bar{\nu}_{\mu}$ \\
\cline { 2 - 5 }$a_{0}$ & 2.7 & 2.7 & 5.1 & 5.1 \\
\cline { 2 - 5 }$b_{0}$ & 7.7 & 8.7 & 8.3 & 8.3 \\
\cline { 2 - 5 } & \multicolumn{5}{c}{$p p$}
\end{tabular}

\begin{tabular}{|c|c|c|c|c|c|c|}
\hline \multirow{3}{*}{$a_{0}$} & \multicolumn{2}{|l|}{$\nu$} & $\nu$ & $\gamma$ & \multicolumn{2}{|r|}{$\gamma$} \\
\hline & 15.5 & 5.8 & 14.8 & 5.9 & 20.0 & 8.8 \\
\hline & 8.0 & 5.3 & 7.3 & 5.0 & 5.8 & 4.0 \\
\hline
\end{tabular}

Table 1. Parameters $a_{0}$ and $b_{0}$ obtained from the EPOS-LHC simulation for the yield of each neutrino species in $p p$ collisions (left) and for the total yield of neutrinos and gammas in proton, $\mathrm{He}$ and $\mathrm{Fe}$ collisions with a proton at rest (right).

type of fit changing $0.1 \mathrm{GeV} \rightarrow 0.2 \mathrm{GeV}$. We find that at high energies Eq. (3.1) provides surprisingly precise fits for all the yields in proton, helium and even iron collisions. The two parameters in the fit change logarithmically with the energy according to

$$
\begin{aligned}
& a=a_{0}\left(1+0.073 \ln \frac{E}{10^{6} \mathrm{GeV}}+0.0070 \ln ^{2} \frac{E}{10^{6} \mathrm{GeV}}\right) \\
& b=b_{0}\left(1+0.020 \ln \frac{E}{10^{6} \mathrm{GeV}}+0.0018 \ln ^{2} \frac{E}{10^{6} \mathrm{GeV}}\right) .
\end{aligned}
$$

In Table 1-left we list the numerical values of $a_{0}$ and $b_{0}$ that fit the yield of (anti)neutrinos of each flavor in $p p$ collisions, whereas in Table 1-right we provide our best fit to the total neutrino and gamma yields for different CR primaries. Analogous parametrizations have been obtained, for example, by Kamae et al. [28], who separate diffractive and non-diffractive contributions, or by Kelner and collaborators [29]. Our EPOS-LHC simulations include neutrinos and gammas from any unstable particle produced (directly or through a complete set hadronic resonances) in these collisions: not only the leading contribution from pions discussed in these references but also from kaons, as well as etas, omegas, neutrons, etc. This may slightly change the spectrum or, for example, also the ratio of flavors in the neutrino yields. In addition, our two parameter fit works well also in the collisions of heavy nuclei (it is straightforward to interpolate the values of $a$ and $b$ for any nucleus between helium and iron).

Repeating the simulations with SIBYLL we obtain very similar results. For example, at $10^{6} \mathrm{GeV}$ we obtain a multiplicity (the 0-moment of the yield) of $44 \nu_{e}+\bar{\nu}_{e}, 83 \nu_{\mu}+\bar{\nu}_{\mu}$ and 47 gammas with EPOS-LHC versus 41, 83 and 50 with SIBYLL, respectively. For the 1.7-moment, we obtain identical results for $Z_{\nu_{\mu} p}^{(1.7)}+Z_{\bar{\nu}_{\mu} p}^{(1.7)}$ but $9 \%$ and $7 \%$ larger values with EPOS-LHC for $Z_{\nu_{e} p}^{(1.7)}+Z_{\bar{\nu}_{e} p}^{(1.7)}$ and $Z_{\gamma p}^{(1.7)}$, respectively. In our calculation of the galactic fluxes we will take the yields from EPOS-LHC and use SIBYLL to estimate the uncertainty. These hadronic simulators do not provide distributions at $E<50 \mathrm{GeV}$; we have checked, however, that the yields obtained with GHEISHA agree reasonably well (the differences in the $Z$-moments are within a $15 \%$ ) with the values provided by the fit in the $1-50 \mathrm{GeV}$ interval of energy. Therefore, we will use our fit in the whole $1-10^{8} \mathrm{GeV}$ interval of kinetic energies.

As for the different approximations discussed in the previous section,

- The dependence of the yields on the $(\mathrm{H}$ or $\mathrm{He})$ target that fragments the $\mathrm{CR}$ is small. We find that $Z_{\nu p}^{(1.7)}$ is just a factor of $c_{T} \approx 1.02$ larger when the target is a He nucleus instead of a proton. The presence of $1 \mathrm{~g}$ of He per $3 \mathrm{~g}$ of $\mathrm{H}$ in the IS medium can then be accounted for by the factor

$$
F_{A}=\frac{3}{4}+\frac{c_{T}}{16} \frac{\sigma_{A \mathrm{He}}}{\sigma_{A p}}
$$


that corrects the $\left(3+4^{-1 / 3}\right) / 4$ factor in Eq. (2.7). For the three CR primaries that we will consider we obtain $F_{p} \approx 0.92, F_{\mathrm{He}} \approx 0.90$ and $F_{\mathrm{Fe}} \approx 0.86$.

- At $\mathrm{CR}$ energies above $1 \mathrm{TeV}$ the $A p$ cross section is not constant but a power law:

$$
\sigma_{A p}(E)=\sigma_{A p}^{0}\left(\frac{E}{1 \mathrm{GeV}}\right)^{\beta_{A}} .
$$

In $p p$ collisions using EPOS-LHC we obtain $\beta_{p}=0.082$ and $\sigma_{p p}^{0}=17.7 \mathrm{mb}$, whereas in $\mathrm{He} p$ and $\mathrm{Fe} p$ collisions we find $\beta_{\mathrm{He}}=0.062, \sigma_{\mathrm{He} p}^{0}=60.5 \mathrm{mb}, \beta_{\mathrm{Fe}}=0.026$ and $\sigma_{\mathrm{Fe} p}^{0}=551 \mathrm{mb}$. This energy dependence will change the spectral index of the galactic neutrinos from $\alpha_{A}$ to $\alpha_{A}-\beta_{A}$. For this reason, in our calculation we will separate the contributions of each component in the CR flux (i.e., we will not use the approximations 1, 3 and 5 discussed in the previous section).

- We will assume a (position-dependent) CR flux of type $\Phi_{A}(E, \vec{r})=g(\vec{r}) \Phi_{A}^{0}(E)$, where $g(\vec{r})$ is the ratio between the flux at position $\vec{r}$ in our galaxy and the flux $\Phi_{A}^{0}(E)$ that we observe at the Earth $(r=0)$. Eq. (2.4) becomes then

$$
\Phi_{\nu}\left(E, \vec{u}_{r}\right)=\sum_{A} \frac{F_{A}}{m_{p}} \int_{0}^{\infty} \mathrm{d} r g(\vec{r}) \rho_{I S}(\vec{r}) \int_{0}^{1} \mathrm{~d} x \sigma_{A p}(E / x) \Phi_{A}^{0}(E / x) x^{-1} f_{A}^{\nu}(x, E / x) .
$$

This is the final equation that we will use to find the neutrino and the gamma-ray fluxes. The sum runs over the different species $A$ in the CR flux, while the effect of the He fraction present in the IS matter is encapsulated in the coefficients $F_{A}$. The energy dependence in this equation simplifies if one assumes that both the CR fluxes and the cross sections follow unbroken power laws and that the yields are energy independent:

$$
\Phi_{\nu}\left(E, \vec{u}_{r}\right)=\sum_{A} \frac{F_{A} \sigma_{A p}^{0} C_{A}}{m_{p}} E^{-\left(\alpha_{A}-\beta_{A}\right)} \int_{0}^{\infty} \mathrm{d} r g(\vec{r}) \rho_{I S}(\vec{r}) \int_{0}^{1} \mathrm{~d} x x^{\alpha_{A}-\beta_{A}-1} f_{A}^{\nu}(x),
$$

where $\Phi_{A}^{0}(E)=C_{A} E^{-\alpha_{A}}$ is the flux of CR nuclei type $A$ at the Earth. We will compare the results provided by this expression with the more general ones from Eq.(3.6).

\section{Angular dependence of the fluxes}

The integral

$$
\tilde{X}_{\nu}\left(\vec{u}_{r}\right) \equiv \int_{0}^{\infty} \mathrm{d} r g(\vec{r}) \rho_{I S}(\vec{r})
$$

that appears in Eq. (3.6) provides all the angular dependence of the galactic neutrino fluxes. Basically, $\tilde{X}_{\nu}\left(\vec{u}_{r}\right)$ is the depth of IS matter along the direction $\vec{u}_{r}$ corrected by the CR density at each point relative to the one that we observe at the Earth. The analogous expression for high-energy gamma rays will include an attenuation factor $\eta(E, \vec{r})$ from gamma-ray collisions with the CMB or with infrared galactic light [30]:

$$
\tilde{X}_{\gamma}\left(E, \vec{u}_{r}\right) \equiv \int_{0}^{\infty} \mathrm{d} r g(\vec{r}) \eta(E, \vec{r}) \rho_{I S}(\vec{r}) .
$$

In particular, the mean free path for the conversion of a $E=0.5-5 \mathrm{PeV}$ gamma ray into an $e^{+} e^{-}$pair through the collision with a CMB photon is around $10 \mathrm{kpc}$, whereas if the gamma 
travels near the galactic center (at radial distances below $4 \mathrm{kpc}$ ) it may experience the same type of conversion at energies 10 times smaller.

For the IS matter in our galaxy, we will take a total mass of $4.6 \times 10^{11} M_{\odot}$, which is $13 \%$ of all its baryonic mass. We will distribute this mass in the thin disk, the thick disk and the galactic halo according the basic scheme described in [31]. The thin disk reaches up to $R \approx 35 \mathrm{kpc}$, with a radial scale $R_{n}=3.15 \mathrm{kpc}$ and a vertical scale height $h_{t}$ that increases with $R$ :

$$
\rho_{t}(R, z)=\left\{\begin{array}{l}
m_{p} n_{t} \exp \left(-\frac{R-R_{\odot}}{R_{n}}-\frac{z \ln 2}{h_{t}(R)}\right) R>7 \mathrm{kpc} \\
m_{p} n_{t} \exp \left(-\frac{7-R_{\odot}}{R_{n}}-\frac{z \ln 2}{h_{t}(R)}\right) \quad R<7 \mathrm{kpc},
\end{array}\right.
$$

where $n_{t}=1.5 \mathrm{~cm}^{-3}, R_{\odot}=8.4 \mathrm{kpc}, h_{t}(R)=h_{t 0} e^{\frac{R-R_{0}}{R_{0}}}, h_{t 0}=0.15 \mathrm{kpc}$ and $R_{0}=9.8 \mathrm{kpc}$. The thick disk has a similar structure but a different density and scale height: $n_{T}=0.15$ $\mathrm{cm}^{-3}$ and $h_{T 0}=0.4 \mathrm{kpc}$, respectively. Finally, we will take a $60 \mathrm{kpc}$ halo with spherical symmetry and a large radial scale:

$$
\rho_{h}(R, z)=m_{p} n_{h} \exp \left(-\frac{\sqrt{R^{2}+z^{2}}-R_{\odot}}{r_{h}}\right),
$$

with $n_{h}=0.001 \mathrm{~cm}^{-3}$ and $r_{h}=12 \mathrm{kpc}$.

For the galactic CR density, we will modify the simple but effective leaky-box model, that assumes a constant density in the galactic disk, by correlating it with the mean magnetic field strength at each point in the disk and the halo. As we have argued in the introduction, the CR spectral index $\alpha_{0}=2.0-2.2$ at the sources is changed into the one we see, $\alpha=2.6-2.7$, by the propagation through the regular and turbulent magnetic fields in the IS medium. The extra suppression of $\propto E^{-\beta}$ with $\beta \approx 0.5$ would reflect that higher energies have a larger diffusion coefficient and a shorter time of escape from our galaxy (a position-dependent coefficient is used, for example, by the CR propagation code DRAGON [32]). Since all magnetic effects depend on $R / B \approx E /(Z e B)$, the flux of a very diffused $C R$ gas should scale

$$
\frac{\Phi_{A}(E, \vec{r})}{\Phi_{A}(E, 0)}=g(\vec{r}) \approx\left(\frac{B(\vec{r})}{B_{0}}\right)^{0.5}
$$

independently of the position of the sources. Eq. (4.5) expresses that CRs stay longer in regions of the galaxy with stronger magnetic fields and, as a consequence, their density in those regions is larger. We will assume that in the disk the magnetic field decays exponentially with a radial scale $R_{B}=8.5 \mathrm{kpc}[33]: B(R)=B_{0} e^{-\left(R-R_{\odot}\right) / R_{B}}$ with $B_{0}=4 \mu \mathrm{G}$, and that in the halo it has a constant value around $B_{h}=1 \mu \mathrm{G}$. Our hypothesis implies then that the $\mathrm{CR}$ density in the galactic center is 1.6 times larger than the one we observe here, whereas in the halo it is a factor of 0.5 smaller.

Fig. 2 summarizes our results for the angular distribution of the fluxes. The plot on the left applies to neutrinos of any energy and gamma rays with $E_{\gamma}<50 \mathrm{TeV}$. It gives a largest effective depth of $0.302 \mathrm{~g} / \mathrm{cm}^{2}$ from the galactic center at R.A. $=-93^{\circ}$ and $\delta=-29^{\circ}$ and just $0.0019 \mathrm{~g} / \mathrm{cm}^{2}$ from the darkest point, at R.A. $=-170^{\circ}$ and $\delta=29^{\circ}$. The average depth is $\left\langle\tilde{X}_{\nu}\right\rangle=0.0080 \mathrm{~g} / \mathrm{cm}^{2}$. The plot on the right refers to photons of $0.5-5 \mathrm{PeV}$, which experience a maximum attenuation by the CMB. $\tilde{X}_{\gamma}$ varies between $0.137 \mathrm{~g} / \mathrm{cm}^{2}$ and $0.0018 \mathrm{~g} / \mathrm{cm}^{2}$, 

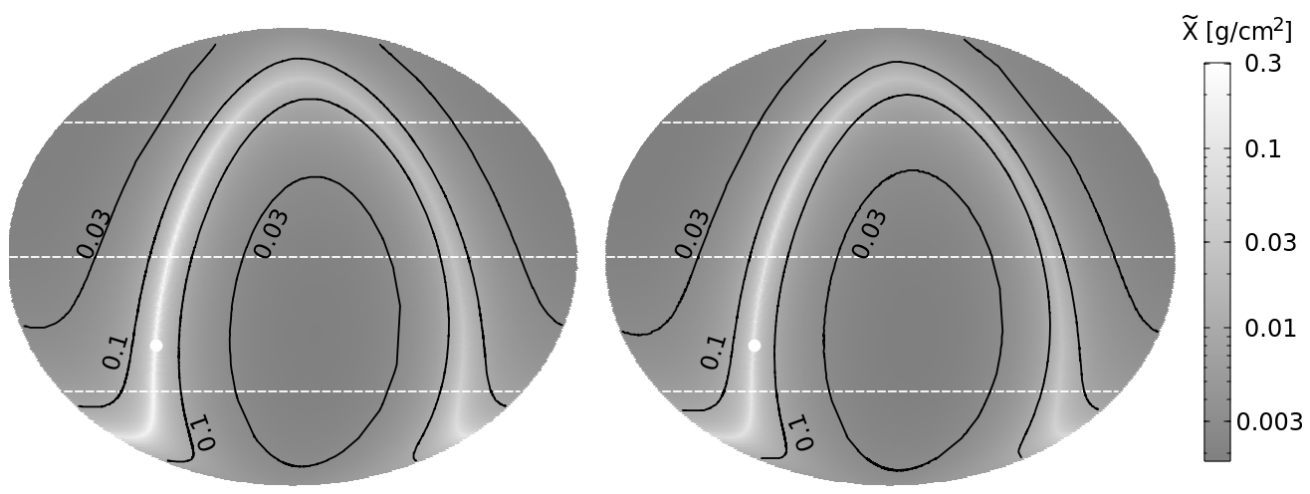

Figure 2. Left. Effective depth along the different directions (equatorial coordinates). We indicate with dashes the declinations $\delta=0^{\circ}, \pm 45^{\circ}$ and with a white dot the galactic center. Right. Effective depth for $0.5-5 \mathrm{PeV}$ photons (it includes the attenuation by the $\mathrm{CMB}$ ).
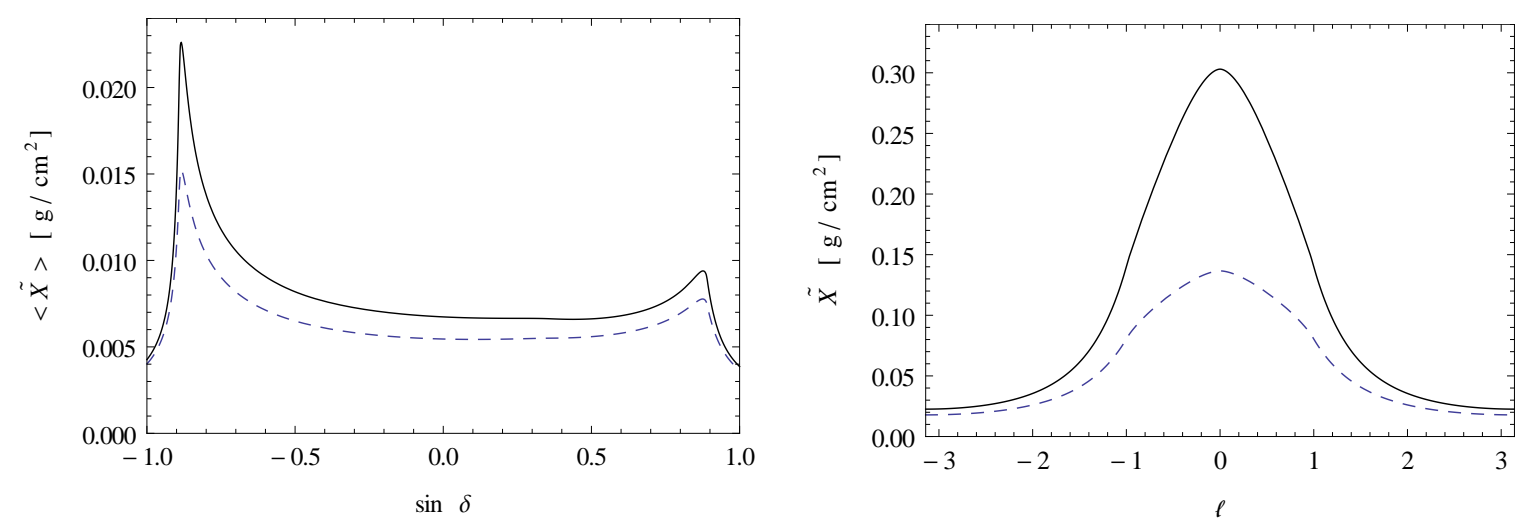

Figure 3. Left. Effective depth at different declinations averaged over the R.A. for neutrinos (solid) and for $0.5-5 \mathrm{PeV}$ gamma rays (dashes). Right. Effective depth of the galactic disk (latitude $b=0$ ) at different longitudes $\ell$.

with an average value of $0.0064 \mathrm{~g} / \mathrm{cm}^{2}$. In Fig. 3-left we plot the average depth for each declination (i.e., we have integrated over the R.A. and divided by $2 \pi$ ), whereas Fig. 3 -right gives the depth of the galactic disc at different longitudes $\ell$. We deduce, for example, that the ratio of positive versus negative declinations is approximately $3: 4$. Neglecting the attenuation of the neutrino flux by the Earth, this will also be the approximate ratio of upgoing versus downgoing neutrinos of galactic origin reaching IceCube.

\section{$5 \quad$ Neutrino and gamma-ray fluxes}

We can finally give the neutrino and gamma-ray fluxes from CR collisions with IS matter. At energies below the knee we have separated the proton and the helium contributions (see the fluxes in Eqs. $(1.1,1.2)$ ). Since at $E \leq 10 \mathrm{GeV}$ the effects of the heliosphere on the CR flux are important, in the $1-10 \mathrm{GeV}$ interval we have considered two possibilities: an unbroken power law (i.e., the same spectral index $\alpha$ that holds at higher energies) or its reduction in one unit (which gives the approximate CR flux that we see at low energies). At $E>E_{\text {knee }}$ 

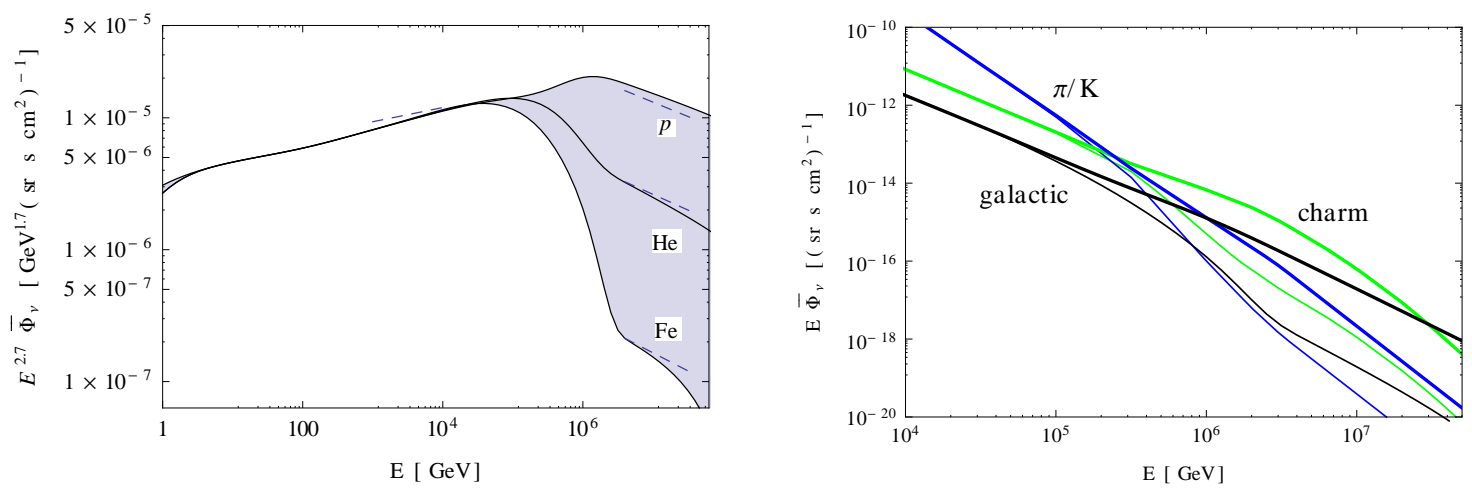

Figure 4. Left. Average (all directions) $\nu$ flux reaching the Earth. The shaded region corresponds to different $\mathrm{CR}$ compositions ( $p, \mathrm{He}$ or $\mathrm{Fe}$ ) at $E>E_{\text {knee }}$. Right. Comparison with the atmospheric $\nu$ fluxes from pion and kaon decays and from forward charm decays. For each contribution, we plot the $\nu$ flux for a proton (thick line) or an iron (thin line) CR composition at $E>E_{\text {knee }}$.

we have studied different CR compositions: proton, He and Fe. A mixed composition can be easily deduced by combining these three possibilities.

In Fig. 4 -left we give the neutrino flux ( $\nu$ plus $\bar{\nu}$ of the three flavors) obtained from Eq. (3.6). It is the flux averaged over all the directions that reach the Earth. The shaded regions reflect the uncertainty in the low-energy $\mathrm{CR}$ flux and in the $\mathrm{CR}$ composition at $E>E_{\text {knee. }}$ In dashes we provide the result derived from the expression in Eq. (3.7). Below $E_{\text {knee }}$ this approximate result is [in $\left.\left(\mathrm{cm}^{2} \mathrm{~s} \mathrm{sr} \mathrm{GeV}\right)^{-1}\right]$

$$
\bar{\Phi}_{\nu}=3.7 \times 10^{-6}\left(\frac{E}{\mathrm{GeV}}\right)^{-2.617}+0.9 \times 10^{-6}\left(\frac{E}{\mathrm{GeV}}\right)^{-2.538},
$$

where the first and second terms correspond to the proton and the He contributions, respectively. The different spectral indexs are related to the different energy dependence of their cross sections. At energies above $E_{\text {knee }}$ Eq. (3.7) implies fluxes

$$
\bar{\Phi}_{\nu}= \begin{cases}4.4 \times 10^{-4}\left(\frac{E}{\mathrm{GeV}}\right)^{-2.918} & \text { (proton) }, \\ 1.2 \times 10^{-4}\left(\frac{E}{\mathrm{GeV}}\right)^{-2.938} & \text { (helium) } \\ 1.3 \times 10^{-5}\left(\frac{E}{\mathrm{GeV}}\right)^{-2.974} & \text { (iron) } .\end{cases}
$$

In Fig. 4-right we compare this galactic flux with the atmospheric one. We see that the conventional neutrino flux from light meson decays is larger than the galactic one for all energies below $1 \mathrm{PeV}$. At $E \approx 250 \mathrm{TeV}$ the neutrino flux from forward charm decays [34, 35] would dominate both the conventional and the galactic fluxes. Notice that the atmospheric $\nu$ flux also has a strong dependence with the CR composition: it scales like the all-nucleon flux, i.e., like $1 / A$ at $E>E_{\text {knee }}$.

We may as well compare our results with the ones obtained by other authors. For example, the galactic diffuse flux at $10-100 \mathrm{TeV}$ deduced with a GALPROP simulation (model ${ }^{S} S^{Z} 4^{R} 20^{T} 150^{C} 5$ ) in [22] is a $25 \%$ smaller than the one we have found here, a difference that is within the uncertainty in our (or any) calculation.

The relative frequency of the different neutrino and antineutrino flavors in this flux is proportional to their $Z$-moment of order $\alpha_{A}-\beta_{A}-1 \approx 1.7$. For proton primaries, before $\nu$ 

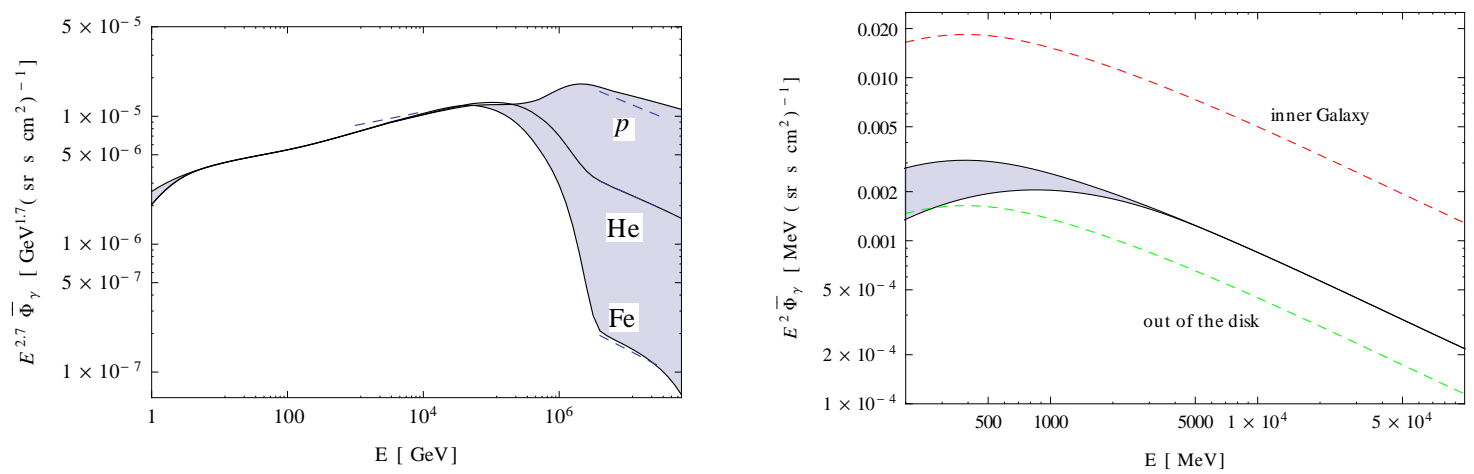

Figure 5. Left. Average gamma-ray flux from hadron fragmentation (it does not include leptonic gamma rays). We plot with dashes the approximate power laws deduced with the $Z$-moment method. Right. Average gamma-ray flux at low energies. We provide the flux in from the inner Galaxy region $\left(-8^{\circ} \leq b \leq+8^{\circ}, 100^{\circ} \leq \ell \leq 260^{\circ}\right)$ and from outside the galactic disk $\left(|b| \geq 8^{\circ}, 0^{\circ} \leq \ell \leq 360^{\circ}\right)$ for an unbroken power law in the low-energy CR flux.

oscillations we find

$$
\left(\nu_{e}: \nu_{\mu}: \nu_{\tau}: \bar{\nu}_{e}: \bar{\nu}_{\mu}: \bar{\nu}_{\tau}\right)=\frac{1}{6}(1.29: 1.89: 0: 0.87: 1.95: 0),
$$

whereas in helium or iron collisions the relative $\nu$ and $\bar{\nu}$ abundances are closer:

$$
\left(\nu_{e}: \nu_{\mu}: \nu_{\tau}: \bar{\nu}_{e}: \bar{\nu}_{\mu}: \bar{\nu}_{\tau}\right)=\frac{1}{6}(1.11: 1.96: 0: 1.06: 1.87: 0) .
$$

This gives, in both cases, a 1.08:1.92 electron to muon ratio, which is a $10 \%$ larger than the 1:2 ratio usually assumed from pion decays. The distances that these galactic neutrinos travel to reach the Earth are much larger than their typical oscillation length. Taking $\theta_{12}=33^{\circ}$, $\theta_{23}=49^{\circ}, \theta_{13}=8^{\circ}$ and $\delta_{\mathrm{CP}}=0$ [36], the probability for a flavor transition is then

$$
P_{\alpha \beta}=\sum_{j}\left|U_{\alpha j}\right|^{2}\left|U_{\beta j}\right|^{2}=\left(\begin{array}{lll}
0.549 & 0.222 & 0.229 \\
0.222 & 0.412 & 0.366 \\
0.229 & 0.366 & 0.405
\end{array}\right),
$$

with $U_{\alpha j}$ the PMNS matrix. For proton and $\mathrm{He} / \mathrm{Fe}$ primaries, respectively, the final frequency of each flavor after oscillations is

$$
\left(\nu_{e}: \nu_{\mu}: \nu_{\tau}: \bar{\nu}_{e}: \bar{\nu}_{\mu}: \bar{\nu}_{\tau}\right)=\frac{1}{6}(1.13: 1.07: 0.99: 0.91: 0.99: 0.91)
$$

and

$$
\left(\nu_{e}: \nu_{\mu}: \nu_{\tau}: \bar{\nu}_{e}: \bar{\nu}_{\mu}: \bar{\nu}_{\tau}\right)=\frac{1}{6}(1.04: 1.06: 0.97: 1.00: 1.00: 0.93)
$$

As for the gamma-ray flux, our results are summarized in Fig. 5. The approximate expression deduced with the $Z$-moment method at energies below the knee is $\left[\mathrm{in}\left(\mathrm{cm}^{2} \mathrm{~s} \mathrm{sr} \mathrm{GeV}\right)^{-1}\right.$ ]

$$
\bar{\Phi}_{\gamma}=3.4 \times 10^{-6}\left(\frac{E}{\mathrm{GeV}}\right)^{-2.617}+0.8 \times 10^{-6}\left(\frac{E}{\mathrm{GeV}}\right)^{-2.538},
$$


that includes a 0.93 attenuation factor from collisions with infrared light in the center of the galaxy [37]. At higher energies gamma-ray interactions with the CMB increase the suppression to 0.80 , implying

$$
\bar{\Phi}_{\gamma}= \begin{cases}4.2 \times 10^{-4}\left(\frac{E}{\mathrm{GeV}}\right)^{-2.918} & \text { (proton), } \\ 1.1 \times 10^{-4}\left(\frac{E}{\mathrm{GeV}}\right)^{-2.938} & \text { (helium), } \\ 1.2 \times 10^{-5}\left(\frac{E}{\mathrm{GeV}}\right)^{-2.974} & \text { (iron) } .\end{cases}
$$

In Fig. 5-right we give the low-energy flux averaged over different galactic regions. At these energies the gamma-ray flux observed by Fermi-LAT [38] includes several components, being the diffuse flux from galactic CR interactions obtained here the dominant one. We may compare our result with their estimate using a GALPROP simulation; for example, in the inner galactic region $\left(-8^{\circ} \leq b \leq+8^{\circ}\right.$ and $\left.100^{\circ} \leq \ell \leq 260^{\circ}\right)$ at $E \leq 1 \mathrm{GeV}$ our gamma-ray flux is very similar to the one they obtain [38] with GALPROP (model ${ }^{S} S^{Z} 4^{R} 20^{T} 150{ }^{C} 5$ ), but at $E=100 \mathrm{GeV}$ ours is $20 \%$ larger. These differences are within the expected uncertainties; incidentally, at 10-100 GeV Fermi-LAT predicts a gamma-ray flux from the inner galactic region that is below the one they observe.

\section{Summary and discussion}

Charged cosmic rays are the only known source of high-energy neutrinos and gamma rays. In addition to the Earth's atmosphere, in our galaxy one may distinguish two different environments where these neutral particles are produced: the dense regions where CRs are accelerated (point-like sources), and the IS space where CRs are trapped for several million years (diffuse flux). A precise characterization of the diffuse flux of galactic neutrinos and gamma rays from CR collisions with IS matter is necessary for the identification of astrophysical sources [39] or in the search for other possible sources, like the annihilation of dark-matter particles.

Here we have attempted a calculation of these fluxes that covers the whole energy spectrum of interest. We have paid special attention to the yields of neutrinos and gamma rays from CR fragmentation at different energies. In particular, we provide a two-parameter fit that gives an accurate description (within the 15\%) for the moments of order 0 (total number of particles), 1 (fraction of energy taken by these particles) and 2 (dictates the total highenergy fluxes) of the neutrino and photon distributions obtained with different simulators (the results with EPOS-LHC, SIBYLL or GHEISHA may differ in a 15\%).

In our study we have assumed a particular model for the distribution of IS matter (hydrogen and helium) in the galaxy. Although this distribution may have a certain degree of uncertainty, it reproduces well the total mass of the galactic gas, which is more constrained and fixes the average neutrino and gamma-ray fluxes in Figs. 4 and 5. We have also assumed a galactic CR density correlated with the mean magnetic field strength in the disk and the halo. This should provide a better description than the constant CR density in the disk with an empty halo used in the usual leaky-box model.

At any rate, we have shown that the two main sources of uncertainty in the galactic neutrino and gamma-ray fluxes are the low-energy CR spectrum out of the heliosphere and the $\mathrm{CR}$ composition at energies beyond $E_{\text {knee. }}$. The first one gives $30 \%$ variations in the gamma-ray flux at energies around $1 \mathrm{GeV}$, where Fermi-LAT has observed an excess from the galactic center [40]. As for the CR composition, the galactic neutrino flux at $5 \mathrm{PeV}$ could be 6 times larger if instead of He the dominant component beyond the CR knee are protons, but it could also be a factor of 0.06 smaller if they are iron nuclei. Notice that this uncertainty 
is larger than the one in the atmospheric flux [35, 41]. The flux of atmospheric neutrinos is proportional to the all-nucleon flux, that at $E>E_{\text {knee }}$ scales like $A^{2} \Phi_{A}(A E) \approx A^{-1}$. In IS space, however, the situation is quite different. First, when a nucleus collides most of the spectator nucleons leave unscattered keeping all their energy; if just one of the nucleons produced pions, the $\nu$ flux would scale like $A \Phi_{A}(A E)$. In addition, in IS space the probability that the CR interacts grows with $A$, which provides an extra factor of $A^{2 / 3}$ and a total neutrino flux proportional to $A^{-4 / 3}$. Our analysis does not use these scaling hypothesis, it is based on EPOS-LHC simulations of the cross sections and neutrino yields that are specific for each primary. The suppresion of $1 / 100$ that we obtain when going from proton to Fe is weaker than the $A^{-4 / 3}=1 / 214$ expected from the previous scaling arguments, but stronger than the $A^{-1}=1 / 56$ present in the atmospheric neutrino flux (see Fig. 4).

We have found that the galactic neutrino flux is small at all IceCube energies. More precisely, we show that at $E_{\nu}<1 \mathrm{PeV}$ it is smaller than the conventional flux from pion and kaon decays, and that at 10-1000 $\mathrm{TeV}$ it is well below the expected flux from forward charm decays (which should dominate the atmospheric neutrino flux at $E_{\nu}>250 \mathrm{TeV}$ ). Of course, the angular distribution and the flavor composition of these fluxes do not coincide, but we estimate that among the 54 high-energy events observed by IceCube during the initial 4-year period, there could be up to 14 events [42] from atmospheric neutrinos while only 1 event would have the galactic origin discussed here.

Although CR propagation and spallation inside our galaxy can be simulated numerically with more sophisticated MonteCarlo codes [17, 22, 32] that include local sources or leptonic gamma rays, we think that the type of simplified analysis presented here may still be useful. Our results seem to agree within a $25 \%$ with complete GALPROP simulations, and the method that we use could be more efficient, for example, to identify the effects of different hypotheses (like changes in the spectral index or in the composition of the CR gas) or to account for the whole domain of the different distributions involved in the calculation (notice that the high-energy yields extend down to $x \approx 10^{-8}$ ). In this sense, we find remarkable that a change in the composition of an otherwise smooth CR spectrum can imply the sharp drop in the flux of galactic neutrinos at $\mathrm{PeV}$ energies shown in Fig. 5. This is an interesting spectral feature that could mimic the ones expected in indirect searches for dark matter.

\section{Acknowledgments}

This work has been supported by MICINN of Spain (FPA2013-47836, FPA2015-68783-REDT, FPA2016-78220 and Consolider-Ingenio MultiDark CSD2009-00064) and by Junta de Andalucía (FQM101). JMC acknowledges a Beca de Iniciación a la Investigación fellowship from the UGR.

\section{References}

[1] K. Greisen, Phys. Rev. Lett. 16 (1966) 748; G. T. Zatsepin and V. A. Kuzmin, JETP Lett. 4 (1966) 78 [Pisma Zh. Eksp. Teor. Fiz. 4 (1966) 114].

[2] D. Hooper, S. Sarkar and A. M. Taylor, Astropart. Phys. 27 (2007) 199.

[3] P. Blasi, Nucl. Phys. Proc. Suppl. 256-257 (2014) 36.

[4] M. Amenomori [Tibet AS-gamma Collaboration], Science 314 (2006) 439; A. A. Abdo et al., Astrophys. J. 698 (2009) 2121.

[5] A. W. Strong and I. V. Moskalenko, Astrophys. J. 509 (1998) 212. 
[6] G. A. de Nolfo et al., Adv. Space Res. 38 (2006) 1558.

[7] A. Shalchi, Nonliner Cosmic Ray Diffusion Theories, Springer-Verlag, 2009.

[8] T.K. Gaisser, Cosmic Rays and Particle Physics, Cambridge University Press, 1990.

[9] M. Boezio and E. Mocchiutti, Astropart. Phys. 39-40 (2012) 95.

[10] Y. S. Yoon et al., Astrophys. J. 728 (2011) 122.

[11] F. Halzen and S. R. Klein, Rev. Sci. Instrum. 81 (2010) 081101.

[12] F. W. Stecker, Astrophys. J. 228 (1979) 919.

[13] V. S. Berezinsky, T. K. Gaisser, F. Halzen and T. Stanev, Astropart. Phys. 1 (1993) 281.

[14] A. W. Strong, I. V. Moskalenko and O. Reimer, Astrophys. J. 537 (2000) 763 Erratum: [Astrophys. J. 541 (2000) 1109].

[15] C. Evoli, D. Grasso and L. Maccione, JCAP 0706 (2007) 003.

[16] K. Murase, M. Ahlers and B. C. Lacki, Phys. Rev. D 88 (2013) 121301.

[17] A. Neronov, D. V. Semikoz and C. Tchernin, Phys. Rev. D 89 (2014) 103002.

[18] J. C. Joshi, W. Winter and N. Gupta, Mon. Not. Roy. Astron. Soc. 439 (2014) 3414 Erratum: [Mon. Not. Roy. Astron. Soc. 446 (2014) 892].

[19] M. Ahlers and K. Murase, Phys. Rev. D 90 (2014) 023010.

[20] M. KachelrieÃß and S. Ostapchenko, Phys. Rev. D 90 (2014) 083002.

[21] S. Dado and A. Dar, JHEAp 9-10 (2016) 9.

[22] M. Ahlers, Y. Bai, V. Barger and R. Lu, Phys. Rev. D 93 (2016) 013009.

[23] T. Pierog, I. Karpenko, J. M. Katzy, E. Yatsenko and K. Werner, Phys. Rev. C 92 (2015) 034906 .

[24] E. J. Ahn, R. Engel, T. K. Gaisser, P. Lipari and T. Stanev, Phys. Rev. D 80 (2009) 094003.

[25] Z. Jakubowski et al. [Crystal Ball Collaboration], Nucl. Instrum. Meth. A 297 (1990) 60.

[26] M. Ajello et al. [Fermi-LAT Collaboration], Astrophys. J. 819 (2016) 44.

[27] P. Lipari, Astropart. Phys. 1 (1993) 195.

[28] T. Kamae, N. Karlsson, T. Mizuno, T. Abe and T. Koi, Astrophys. J. 647 (2006) 692 Erratum: [Astrophys. J. 662 (2007) 779].

[29] S. R. Kelner, F. A. Aharonian and V. V. Bugayov, Phys. Rev. D 74 (2006) 034018 Erratum: [Phys. Rev. D 79 (2009) 039901].

[30] F.A. Aharonian, Very High Energy Cosmic Gamma Radiation, World Scientific, 2004.

[31] P.M.W. Kalberla and J. Kerp, Ann. Rev. Astron. Astrophys. 47 (2009) 27.

[32] C. Evoli, D. Gaggero, D. Grasso and L. Maccione, JCAP 0810 (2008) 018, Erratum: [JCAP 1604 (2016) no.04, E01].

[33] J. L. Han, R. N. Manchester, A. G. Lyne, G. J. Qiao and W. van Straten, Astrophys. J. 642 (2006) 868.

[34] F. Halzen and L. Wille, Phys. Rev. D 94 (2016) 014014.

[35] T. K. Gaisser, EPJ Web Conf. 52 (2013) 09004.

[36] M. C. Gonzalez-Garcia, M. Maltoni and T. Schwetz, JHEP 1411 (2014) 052.

[37] I. V. Moskalenko, T. A. Porter and A. W. Strong, Astrophys. J. 640 (2006) L155.

[38] M. Ackermann et al. [Fermi-LAT Collaboration], Astrophys. J. 750 (2012) 3. 
[39] M. D. Kistler and J. F. Beacom, Phys. Rev. D 74 (2006) 063007.

[40] D. Gaggero, M. Taoso, A. Urbano, M. Valli and P. Ullio, JCAP 1512 (2015) no.12, 056.

[41] P. Lipari, "Establishing the astrophysical origin of a signal in a neutrino telescope," arXiv:1308.2086 [astro-ph.HE].

[42] J. I. Illana, M. Masip and D. Meloni, Astropart. Phys. 65 (2015) 64. 